\title{
Palladium-Catalyzed $\alpha$-Heteroarylation Route to 4-, 5-, 6- and 7-Azaindoles
}
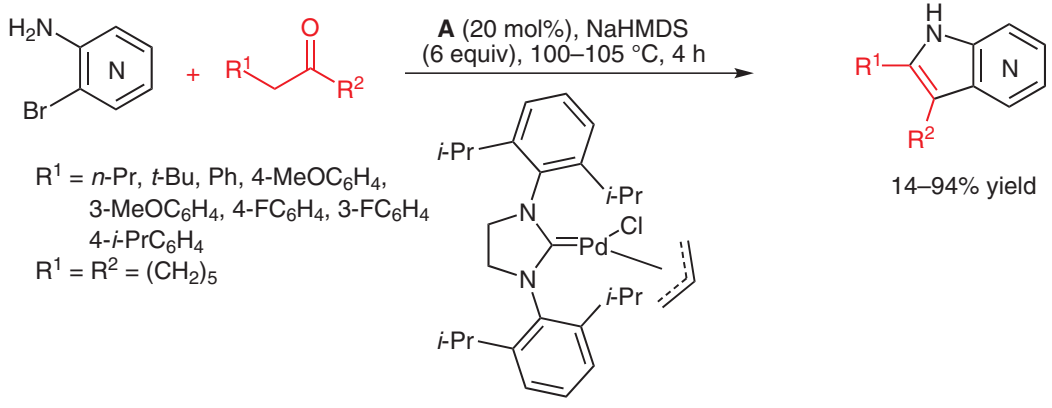

$(\mathrm{SIPr}) \operatorname{Pd}(\operatorname{allyl}) \mathrm{Cl}(\mathbf{A})$

Significance: On their way to lkB kinase $\beta$ inhibitors, BMS scientists developed a convenient route to azaindoles as reported herein. The first approach involving a Sonogashira coupling and subsequent annulation required isolation of intermediates. In this route with no C2-substitution complex mixtures were obtained; furthermore, trying to follow with direct C2-arylation was unsuccessful. Additionally, the palladium-catalyzed enamine Heck coupling was only successful with ethyl pyruvate, and the palladium-catalyzed tandem coupling of gem-dichloroolefins was only successful with substrates having few or no substituents. Therefore, the direct arylation of enolates with 2-amino-3-bromopyridine was explored. After optimization, the use of Nolan's $\mathrm{N}$-heterocyclic carbene catalyst and $\mathrm{Na}$ - or LiHMDS as base resulted in complete conversions. The desired 7 -azaindoles were isolated in moderate to excellent yields. Extension of the methodology to 4-, 5- and 6-azaindoles (and the synthesis of the desired kinase inhibitor) was successful, albeit in lower yields. Both aryl and alkyl ketones were successful in the reaction.
Comment: Azaindoles represent one of the most important recent heterocyclic scaffolds for the development of new protein kinase inhibitors (see Review below) and, as a result, several methods exist for their construction. Regardless, these methods were not amenable for the efficient construction of the particular azaindole the authors desired. The subsequently adapted $\alpha$-heteroarylation method involves the use of simple commercially available starting materials and allows access to all regioisomeric azaindoles, a feat which is achieved by only a few routes (e.g. N. Lachance, M. April, M.-A. Joly Synthesis 2005 , 2571). Although the scope of the ketone component was well studied, the pyridine counterpart was not studied in terms of substituents. Interestingly, six equivalents of base is required and the use of bases other than Na- or LiHMDS afforded incomplete conversions and resulted in significant amounts of ketone self-condensation. The reason for these observations was not rationalized.

Review: J. J. Song et al. Chem. Soc. Rev. 2007, 36, 1120-1132. 